\title{
Declaración: La Sociedad Chilena de Infectología frente a la situación epidemiológica de hepatitis A en Chile
}

\author{
Statement: The Infectious Diseases Society and the \\ epidemiological situation of hepatitis A in Chile
}

\section{Situación epidemiológica de la hepatitis A en Chile a julio de 2003}

El virus de la hepatitis A (VHA) ha sido y sigue siendo un agente endémico en Chile. Varias encuestas serológicas realizadas en los últimos $10 \mathrm{a}$ 15 años, tanto en la Región Metropolitana como en otras zonas del país, han mostrado un desplazamiento progresivo de la seroconversión hacia una mayor edad promedio en que la población adquiere la infección por el VHA. Este fenómeno es característico de comunidades en transición socioeconómica, y consecuencia directa de la mejoría gradual de las condiciones materiales de vida y del acceso a la infraestructura de saneamiento básico por parte de la población. Sin embargo, antes de alcanzar una reducción en las tasas de ataque de la enfermedad, la consecuencia más inmediata de este desplazamiento es la acumulación de escolares, adolescentes y adultos jóvenes susceptibles, terreno propicio para la ocurrencia de ciclos epidémicos en la población general y de brotes circunscritos en localidades o grupos cerrados.

Las fluctuaciones en la incidencia de hepatitis A observadas en Chile en los últimos 25 años son plenamente consistentes y explicables por el fenómeno antes mencionado. En efecto, entre mediados de los años 70 y el año 2000 se registraron tres claros ciclos epidémicos en el país, que alcanzaron una incidencia máxima de 85 a 110 casos por 100.000 habitantes, y mínima cercana a 40 por 100.000 habitantes.

Entre los años 1998 y 2001 las tasas de hepatitis A fluctuaron entre 38 y 40 por 100.000 habitantes, y alrededor de 5.000 casos reportados por año. En el año 2002 se notificaron 11.188 casos, lo que correspondió a una tasa de 71,8 por 100.000 habitantes, representando un incremento de $94 \%$ respecto al año previo. Durante el presente año se han reportado a la semana epidemiológica 29 (julio 14) un total de 6.867 casos. De mantenerse la tendencia actual, se estima que podría alcanzar a 13.000 casos durante 2003 y una tasa cercana a 75 por 100.000 habitantes. Ello significa que, en globo, durante 2003 uno de cada 1.300 chilenos tendría un episodio de infección por VHA con manifestaciones clínicas, siendo probable que estas cifras sean aún mayores, en consideración a que existe una importante (aunque no cuantificada) subnotificación de casos. El riesgo de desarrollar una hepatitis fulminante, la expresión más grave de la enfermedad, es cercano a $0,1 \%$. Es decir, es esperable que ocurran entre 10 y 15 casos de hepatitis fulminante por VHA durante 2003.

El paso de una endemia intermedia a una endemia baja propia de los países industrializados, requiere de una reducción sustantiva de todas las posibles fuentes de infección, especialmente aguas servidas no tratadas y alimentos insuficientemente cocidos. Es dable esperar que esta transición favorable demore varios años, período durante el cual se debieran mantener incidencias similares a las observadas durante los últimos años, con fluctuaciones dependientes de la adherencia de la comunidad a las recomendaciones entregadas por las autoridades para la higiene personal y ambiental, la disponibilidad de agua potable y el avance de las obras de tratamiento de las aguas servidas. 


\section{Antecedentes de la vacuna anti hepatitis $\mathbf{A}$}

Las vacunas anti hepatitis A actualmente disponibles en Occidente son fabricadas con virus inactivados, y en evaluaciones de campo han demostrado ser altamente eficaces (> 90\%) y seguras. El esquema de vacunación consiste en una dosis de vacuna, seguida de un refuerzo al menos 6 meses después.

El seguimiento durante varios años de los títulos de anticuerpos obtenidos en adultos vacunados, ha permitido estimar que éstos se mantienen en niveles protectores por lo menos durante 20 años después de administrado el esquema de dos dosis. Sin embargo, se requerirán seguimientos a largo plazo de niños vacunados en zonas endémicas, para poder afirmar con certeza cuál es la duración de la protección ante la exposición natural al VHA, y determinar con fundamentos la real necesidad de una vacunación de refuerzo y de su periodicidad, para mantener dicha inmunidad a lo largo de toda la vida.

\section{La vacuna anti hepatitis A puede ser utilizada con tres enfoques:}

- Control de brotes: Implica la utilización de la vacuna en grupos circunscritos (ya sea instituciones como colegios, guarderías, hogares de menores, etc, o familias, barrios, campamentos), donde se presenta un brote de hepatitis A. Esta estrategia ha demostrado alta eficacia en el control de los brotes, con resultados similares a los obtenidos con inmunoglobulina normal, teniendo en consideración que esta segunda otorga una protección transitoria. La eficacia de la estrategia de vacunar en los brotes requiere de una detección oportuna de ellos, basado en un sistema de vigilancia epidemiológica eficiente que abarque todo el territorio nacional, de una intervención precoz (antes de los 18 días de haberse iniciado el brote), y de una alta cobertura en los grupos afectados. Esta medida ha sido capaz de reducir la prolongación de un brote epidémico de 18 meses a tan sólo dos meses.

- Vacunación universal: Implica implementar la vacunación anti hepatitis A dentro del Programa Ampliado de Inmunizaciones (PAI) de un país, en edades específicas, con una distribución y coberturas aseguradas para toda la población blanco. En los países donde esta estrategia ha sido implementada, ya sea focalizada a las regiones geográficas con mayores tasas o en forma generalizada, se ha observado una importante reducción del número de casos en pocos años.

- Vacunación de grupos de riesgo: Implica la vacunación exclusiva de los grupos más afectados. Distintas experiencias han demostrado que la sumatoria de estos grupos no alcanza al $20 \%$ del total de una población dada, por lo que la sola vacunación de estos grupos tiene un bajo impacto en la reducción del problema.

\section{Recomendaciones Sociedad Chilena de Infectología}

En base a la información entregada, la Sociedad Chilena de Infectología ha formulado las siguientes recomendaciones inmediatas y de mediano plazo:

\section{Inmediatas}

- Respaldar y colaborar con el Ministerio de Salud de Chile en la implementación de las medidas higiénicas recomendadas para el control de las infecciones entéricas, a través de la educación activa de la comunidad.

- Contribuir al uso efectivo de vacuna en el control de brotes de hepatitis A. Ello requiere de extremar el sistema de vigilancia y notificación, la rápida identificación de áreas donde esté ocurriendo un brote, y disponer de vacunas para inmunizar a la población blanco, la que se deberá definir de acuerdo al lugar de ocurrencia del brote.

- Recomendar a los médicos clínicos la realización de estudio etiológico en todos los casos de hepatitis aguda diagnosticados en el ámbito ambulatorio y hospitalario.

- Recomendar el cumplimiento en forma cabal y oportuna de la norma de Notificación Obligatoria de hepatitis aguda infecciosa, aportando toda la información solicitada en el formulario RMC-14, y complementando la documentación etiológica del caso tan pronto esta esté disponible.

La implementación de las dos primeras medidas contribuirá a disminuir los casos de hepatitis A y adicionalmente otras enfermedades de transmisión entérica, pero no será suficiente para el control definitivo de la enfermedad en el país. La implementación de las tercera y cuarta medidas ayudará a tener un mejor diagnóstico etiológico 
del síndrome de hepatitis aguda y a minimizar la subnotificación de la enfermedad.

\section{De mediano plazo}

- La Sociedad Chilena de Infectología trabajará estrechamente con el Ministerio de Salud, a través del PAI y su comisión asesora, evaluando la posibilidad de implementar la vacunación programática contra la hepatitis A.

- Este programa de vacunación universal, de implementarse, debería mantenerse hasta que el país alcance un nivel homogéneo y suficiente de saneamiento ambiental como para interrumpir la circulación endémica del virus.
- No respaldamos la estrategia de realizar una campaña de vacunación única y focalizada al grupo actualmente más afectado (5 a 15 años). Este tipo de medida es recomendable cuando existe un brote significativo de una infección limitado a un grupo etario, y determinado por razones biológicas propias de esa infección. La hepatitis A en Chile es endémica, puede afectar a diferentes grupos de edades, de diferentes niveles socioeconómicos, y por ende, la vacunación selectiva de grupos etarios aparece como una solución parcial, cuyo efecto es difícil de precisar y que no va a ayudar a solucionar el problema de fondo.

Santiago, Agosto de 2003 